$15^{\text {th }}$ International Conference on

AEROSPACE SCIENCES \& AVIATION TECHNOLOGY,

$\boldsymbol{A S A T}$ - 15 - May 28 - 30, 2013, Email: asat@mtc.edu.eg,

Military Technical College, Kobry Elkobbah, Cairo, Egypt,

Tel: +(202) 24025292 -24036138, Fax: +(202) 22621908

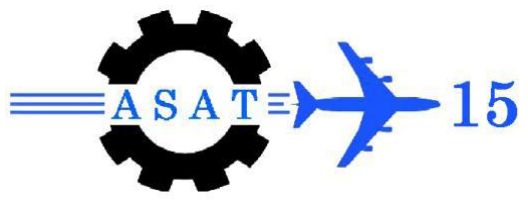

\title{
Computer Aided Reverse Engineering of Machine Parts
}

\author{
S. Khairy ${ }^{*}$, M. K. Shoukry ${ }^{\dagger}$, N.Gad-Allah ${ }^{\ddagger}$ and M. F. Abd El-Samie ${ }^{\S} \S$
}

\begin{abstract}
One of the methods to decrease the technology gap between different countries and to transfer complex technologies is reverse engineering. In Reverse engineering of mechanical parts it becomes necessary to obtain a geometric model of a part either to modify the existing design or to analyze it using schemes such as the Finite Element Method (FEM). The objective of this work is to develop and construct the solid model of an existing machine part by the geometric analysis of three copies of this part. Shape and dimensions of the part are obtained using laser scanner arms and the probing mechanism of a Coordinate-Measuring Machine (CMM). Segmentation \& surface fitting are conducted using Geomagic Studio 12 software and the solid models as well as the workshop drawings are generated on UGS NX 7.5 CAD software. Material analysis and mechanical testing are performed to identify the polymer from which the part is produced. The prototype of this part is constructed Using 3D rapid prototyping machine using the STL file of the produced CAD solid model. The final results of this work are the construction of the complete solid model of the part with all its features and dimensions as well as its material using reverse engineering techniques.
\end{abstract}

Keywords: Computer aided design, reverse engineering, coordinate measuring machine, rapid prototyping.

\section{Introduction}

Reverse engineering is a process of reproducing the geometry of an available physical object. The method of reverse engineering is either manual or computer aided.[1]

Reverse engineering is the opposite of forward engineering. It takes an existing product, and creates a CAD model, for modification or reproduction to the design aspect of the product. It can also be defined as the process of duplicating an existing component by capturing the components physical dimensions. Reverse engineering is usually undertaken in order to redesign the system for better maintainability or to produce a copy of a system without access to the design from which it was originally produced.[2]

\section{Reasons for Reverse Engineering}

- The original manufacturer no longer exists, but a customer needs the product, e.g., aircraft spares required typically after an aircraft has been in service for several years.

- The original manufacturer of a product no longer produces the product, e.g., the original product has become obsolete.

Mech. Eng., Ministry of Military Production, Egypt; saharkhairy@ hotmail.com

† Prof., GUC, Egypt; mohamed.shoukry@guc.edu.eg

* Prof. Modern Academy, Egypt; nabilgadallah@ hotmail.com

$\S \quad$ Egyptian Armed Forces, Egypt, mohamed.fahmy203@ @otmail.com 
- The original product design documentation has been lost or never existed.

- Creating data to refurbish or manufacture a part for which there are no CAD data, or for which the data have become obsolete or lost.

- Inspection and/or Quality Control-Comparing a fabricated part to its CAD description or to a standard item.

- Some bad features of a product need to be eliminated e.g., excessive wear might indicate where a product should be improved.[3].

\section{Procedure of Reverse Engineering}

Reverse engineering typically starts with measuring a physical object to reconstruct a CAD model for applications like redesign, reproduction and quality control, the flow chart shown in figure 1 characterizes the typical procedure of reverse engineering, which consists of five steps:

(1) Data acquisition.

(2) Preprocessing (noise filtering and merging).

(3) Triangulation.

(4) Feature extraction.

(5) Segmentation and surface fitting. [4]

The most critical step of reverse engineering is the segmentation process of the fifth step. The segmentation process splits a triangular mesh into sub-meshes to which an appropriate single surface can be fitted and it seriously affects the quality of the resulting CAD model. To improve the quality of segmentation, it is essential to make use of the features (sharp edges and symmetry planes) extracted in the fourth step.[4]

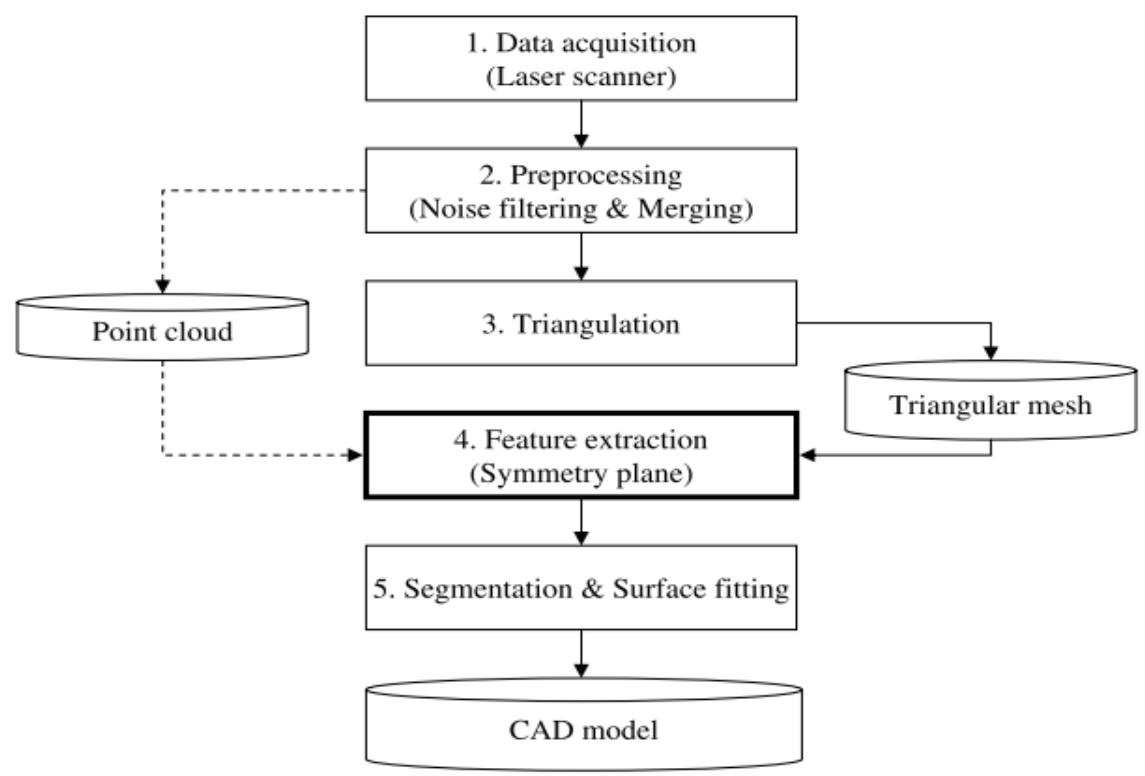

Fig. 1 Typical flow of Reverse Engineering [4]

Computer-aided reverse engineering relies on the use of Computer-aided tools for obtaining the part geometry, identifying its material, improving the design, tooling fabrication, manufacturing planning and physical realization as shown in figure 2. A solid model of the part is the backbone for computer-aided reverse engineering. The model data can be exported from or imported into CAD/CAE/CAM systems using standard formats such as IGES, STL, VDA and STEP. [5] 


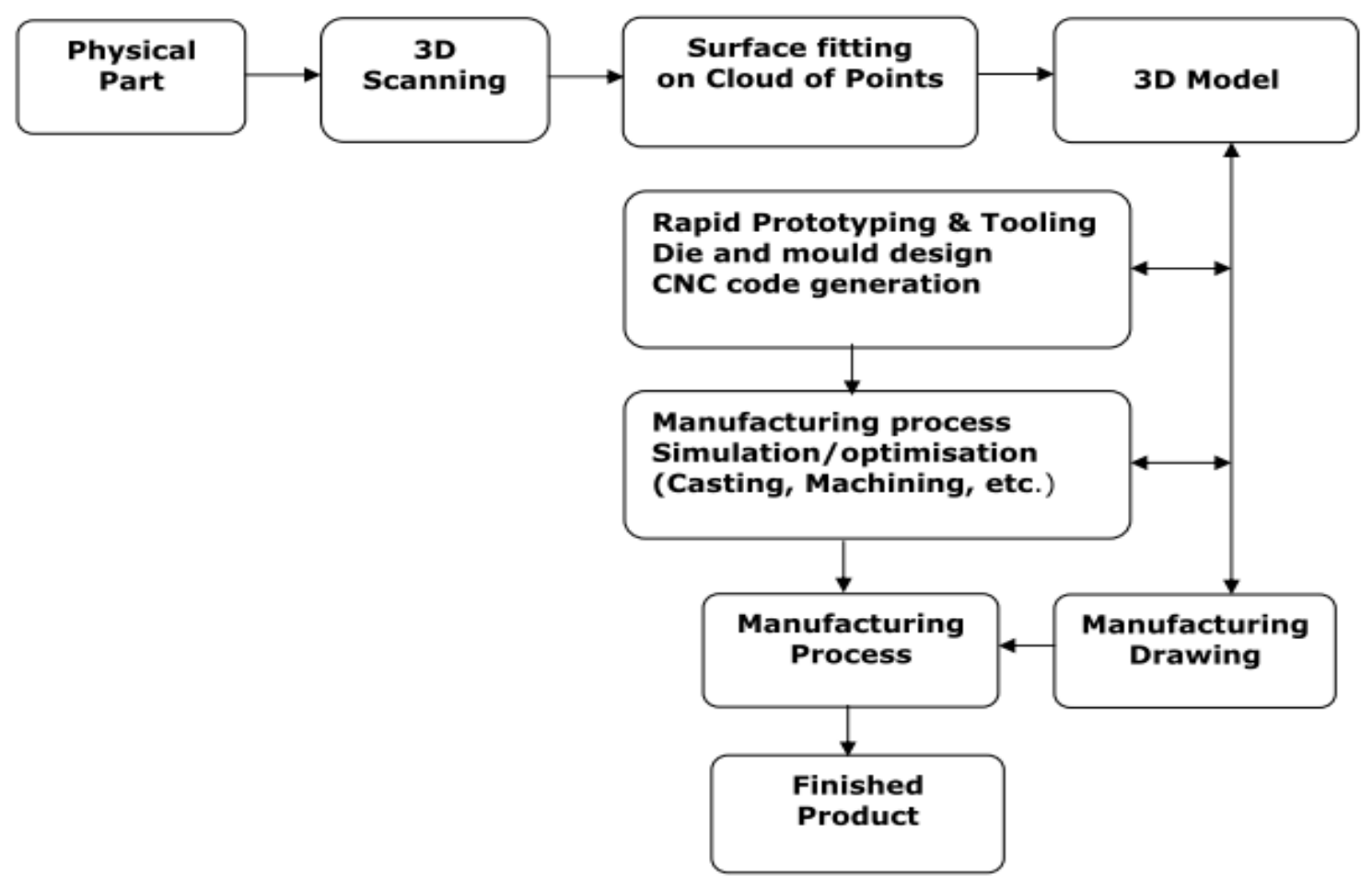

Fig. 2 Computer-aided reverse engineering framework [5]

\section{Experimental and Computer Work}

\subsection{Geometric Analysis}

\subsubsection{Data acquisition by digitization}

At this process, capturing the data of the physical object is carried out by scanning it using Faro-arm laser scanner to obtain a cloud of points representing the required configuration. The following problems were encountered:

- The object was made from a polymeric material and it wasn t possible to fix it using the magnetic table of the arm so the object was fixed vertically on its base using wax.

- Several features like the holes at the two ends of the part were difficult to scan.

- Noises resulted from repeating scan at the same points.

Because the laser beam of the scanner must be orthogonal to the scanned surface, scanning cannot be applied on some surfaces like internal and the base surfaces. This can be seen in the resulted cloud of points as shown in figure (3-A).

Repeating the scan of the object at two different positions by using the Cimcore arm gave two clouds of points which have been assembled using Geomagic Studio 12 software program. The resulted improved cloud of points is shown in figure (3-B).

These steps were repeated for three different copies of the part and resulted in the three clouds of points as shown in figure (3-C) 


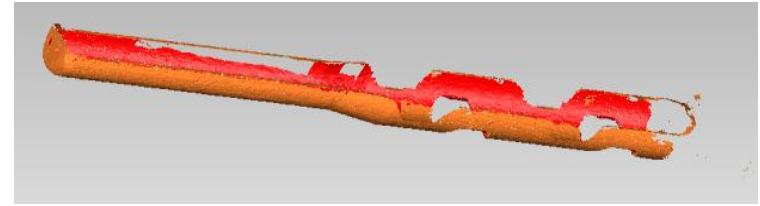

A

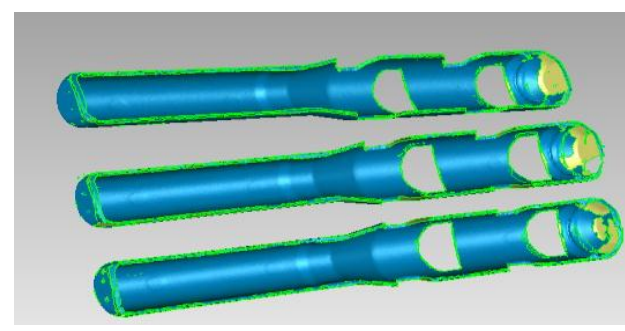

C

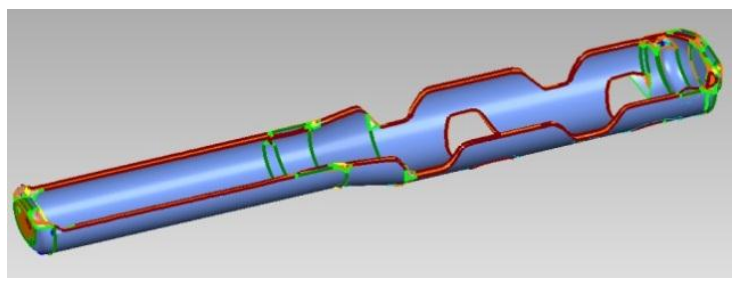

E

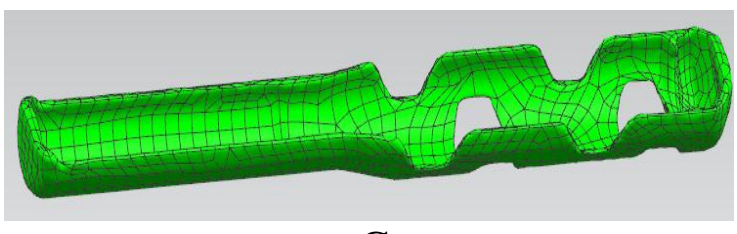

G

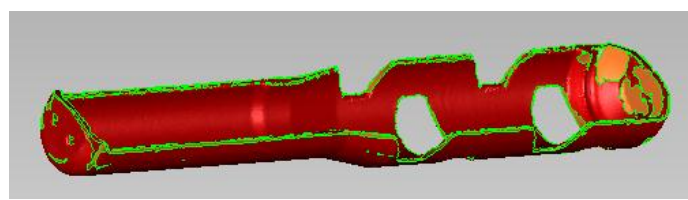

B

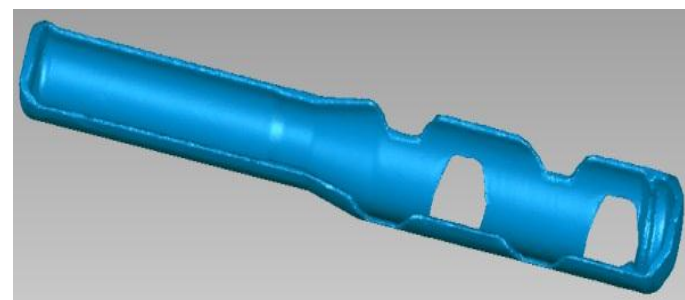

$\mathrm{D}$

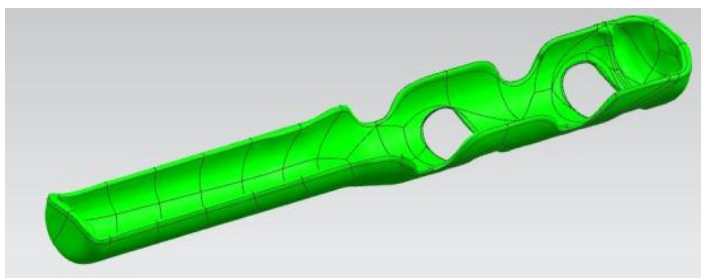

F

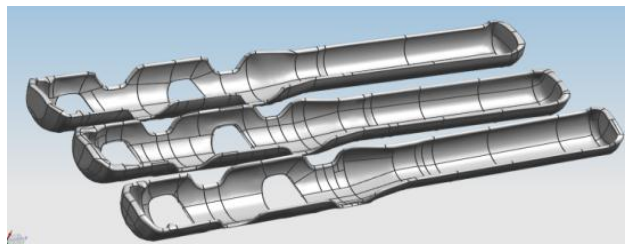

$\mathrm{H}$

Fig. 3 Different laser beam scanning results

\subsubsection{Preprocessing}

Preprocessing was applied on the resulted clouds of points to remove the noises and the redundant points.

\subsubsection{Triangulation}

To convert the clouds of points into polygons with warp command, filling holes, decimate, smooth polygon mesh and repairing the polygon mesh using mesh doctor commands of the software. Figure (3-D) shows the resulted repaired polygons object.

\subsubsection{Feature extraction.}

The purpose of this step is to detect regions and contours and to edit the contours.

\subsubsection{Segmentation and surface fitting.}

This means starting parametric surfacing then fit surfaces and finally classifies connections and fit connections. Figure (3-E) shows the resulted surface part where errors due to filling holes seem to be very large

Starting exact surfacing is following the same procedures as in parametric surfacing to get the resulted surface object shown in figure $(3-\mathrm{F})$. Repeat these steps again to decrease the tolerance between the segments when sewing these segments together. This will produce the 
surface part shown in figure (3-G). To get more accurate surface, polygon object could be cut into small parts and fit surface to these small parts to get the surface object. And these procedures were carried out for the three copies of the object as shown in figure (3-H).

The three exact surface models were imported from Geomagic software program to UGS NX 7.5 CAD software program to convert the surface model parts to solid model parts.

Coordinate measuring machine (CMM) "ACCURA" was then used to get the missing data of the two holes at the two bases of the part and also were selected to check the critical dimensions. The CMM is controlled with Calypso software program.

Eight sections were selected at different distances from the base to measure the required dimensions and Compare the results with the corresponding results obtained from similar sections made on the CAD solid model as shown in figure 4. This comparison is illustrated on Table 1.

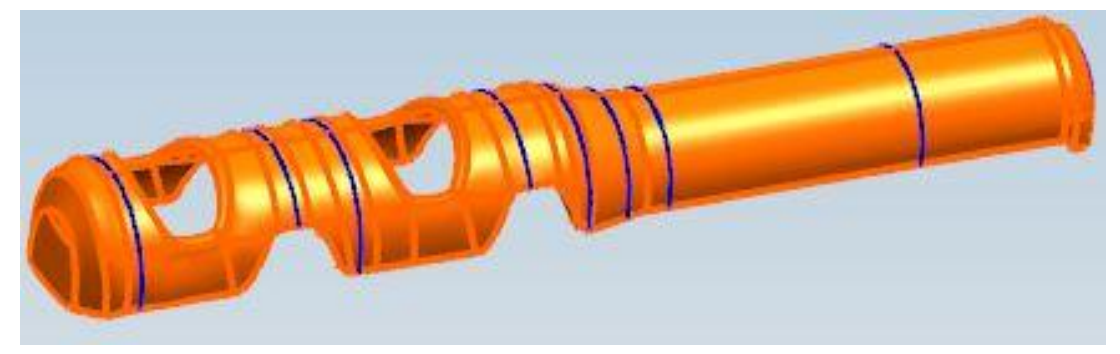

Fig. 4 Eight sections at the CAD solid model

Table 1 Comparison between the original part \& CAD solid models

\begin{tabular}{c|c|c|c|c|c|c|c}
\hline $\begin{array}{c}\text { Z } \\
(\mathrm{mm})\end{array}$ & $\begin{array}{c}\text { CMM S3 } \\
(\mathrm{mm})\end{array}$ & $\begin{array}{c}\text { S3 } \\
(\mathrm{mm})\end{array}$ & ERROR 1 & $\begin{array}{c}\text { S1 } \\
(\mathrm{mm})\end{array}$ & $\begin{array}{c}\text { S2 } \\
(\mathrm{mm})\end{array}$ & $\begin{array}{c}\text { mean of 3 CAD } \\
\text { models }(\mathrm{mm})\end{array}$ & ERROR 2 \\
\hline 44.9926 & 29.125 & 29.662 & $-1.8 \%$ & 29.307 & 29.446 & 29.47166667 & $-1.2 \%$ \\
\hline 44.9976 & 25.467 & 25.218 & $1.0 \%$ & 25.592 & 25.307 & 25.37233333 & $0.4 \%$ \\
\hline 107.9932 & 29.292 & 29.471 & $-0.6 \%$ & 28.479 & 29.208 & 29.05266667 & $0.8 \%$ \\
\hline 108.0025 & 23.866 & 23.795 & $0.3 \%$ & 23.866 & 23.828 & 23.82966667 & $0.2 \%$ \\
\hline 118.9903 & 31.806 & 31.448 & $1.1 \%$ & 31.406 & 32.101 & 31.65166667 & $0.5 \%$ \\
\hline 118.9978 & 27.41 & 27.259 & $0.6 \%$ & 23.511 & 27.708 & 26.15933333 & $4.6 \%$ \\
\hline 129.9906 & 36.954 & 36.471 & $1.3 \%$ & 36.069 & 36.238 & 36.25933333 & $1.9 \%$ \\
\hline 129.9964 & 32.271 & 31.959 & $1.0 \%$ & 30.71 & 31.766 & 31.47833333 & $2.5 \%$ \\
\hline 144.9910 & 38.901 & 38.265 & $1.6 \%$ & 37.073 & 38.201 & 37.84633333 & $2.7 \%$ \\
\hline 144.9989 & 34.335 & 34.189 & $0.4 \%$ & 33.606 & 35.255 & 34.35 & $0.0 \%$ \\
\hline 187.9896 & 38.109 & 39.27 & $-3.0 \%$ & 37.959 & 38.196 & 38.475 & $-1.0 \%$ \\
\hline 188.0031 & 34.292 & 36.193 & $-5.5 \%$ & 34.2437 & 33.834 & 34.7569 & $-1.4 \%$ \\
\hline 202.9888 & 38.39 & 38.898 & $-1.3 \%$ & 37.697 & 37.792 & 38.129 & $0.7 \%$ \\
\hline 203.0036 & 34.202 & 34.524 & $-0.9 \%$ & 33.923 & 34.313 & 34.25333333 & $-0.2 \%$ \\
\hline 242.9832 & 38.989 & 39.354 & $-0.9 \%$ & 39.05 & 39.21 & 39.20466667 & $-0.6 \%$ \\
\hline 244.6645 & 33.566 & 30.95 & $7.8 \%$ & 31.83 & 28.592 & 30.45733333 & $9.3 \%$ \\
\hline$Z$
\end{tabular}

$\mathrm{Z}$ : The height of the section from the base.

CMM S3: CMM measured dimensions of the best scanned copy of the part.

S1, S2, S3: Dimensions of three CAD models.

ERROR 1: \% difference (in dimensions) between the best scanned original copy and it's CAD model.

ERROR 2: \% difference (in dimensions) between the best scanned original copy and the mean of the three CAD models. 
It can be noticed that the difference between the CMM measurements and the mean value of the three CAD models is ranging between $0 \%$ to $9.3 \%$

In order to reduce this difference it was necessary to construct a horizontal plane intersecting the resulted final surface to get the shape of the contour of the part at this plane. Redraw this contour taking into consideration the dimensions measured by the CMM, and then revolve this contour to construct the solid model. All other features were constructed using the information from the surface model and the CMM readings. The final model is shown in figure 5 .

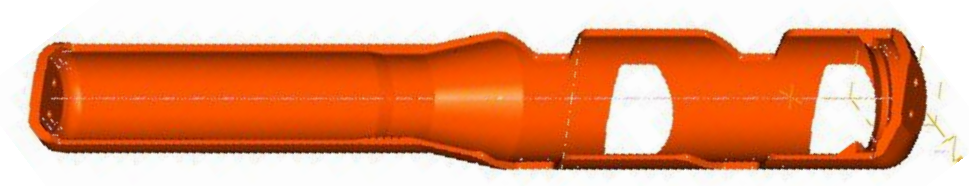

Fig. 5 The final solid model

\subsection{Material Analysis}

\subsubsection{Mechanical properties tests}

Due to the shortage of the material of the scanned part it was decided to perform only tensile and hardness tests.

\section{Tension tests}

Four nonstandard specimens were cut from defected copies of the object, three specimens had a rectangular cross section $2 \times 4 \mathrm{~mm}$ and the fourth was $2 \times 4.5 \mathrm{~mm}$, the length of the specimens was $85 \mathrm{~mm}$. The resulted load- strain curves are shown in figures 6,7 and 8

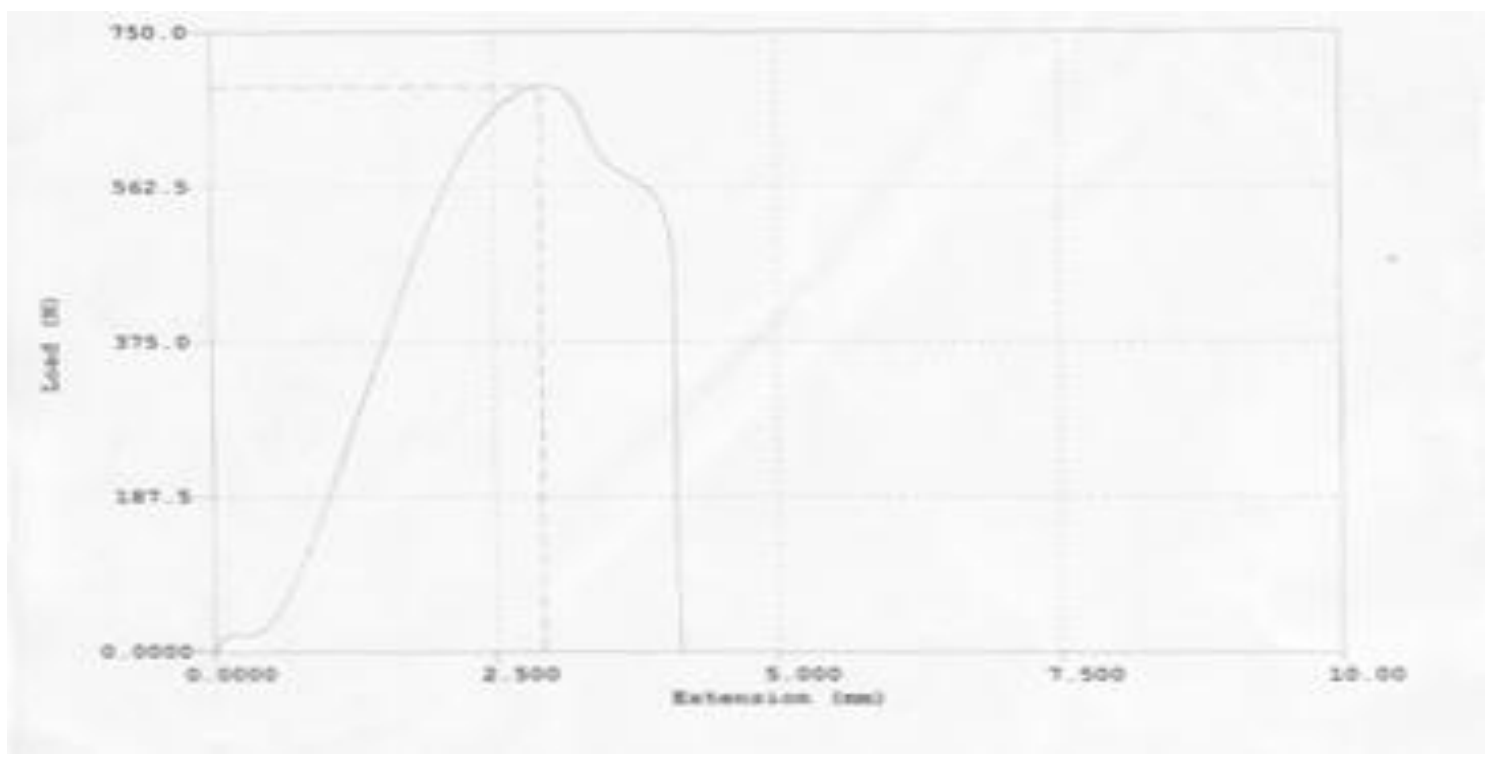

Fig. 6 The resulted load strain curve 1 


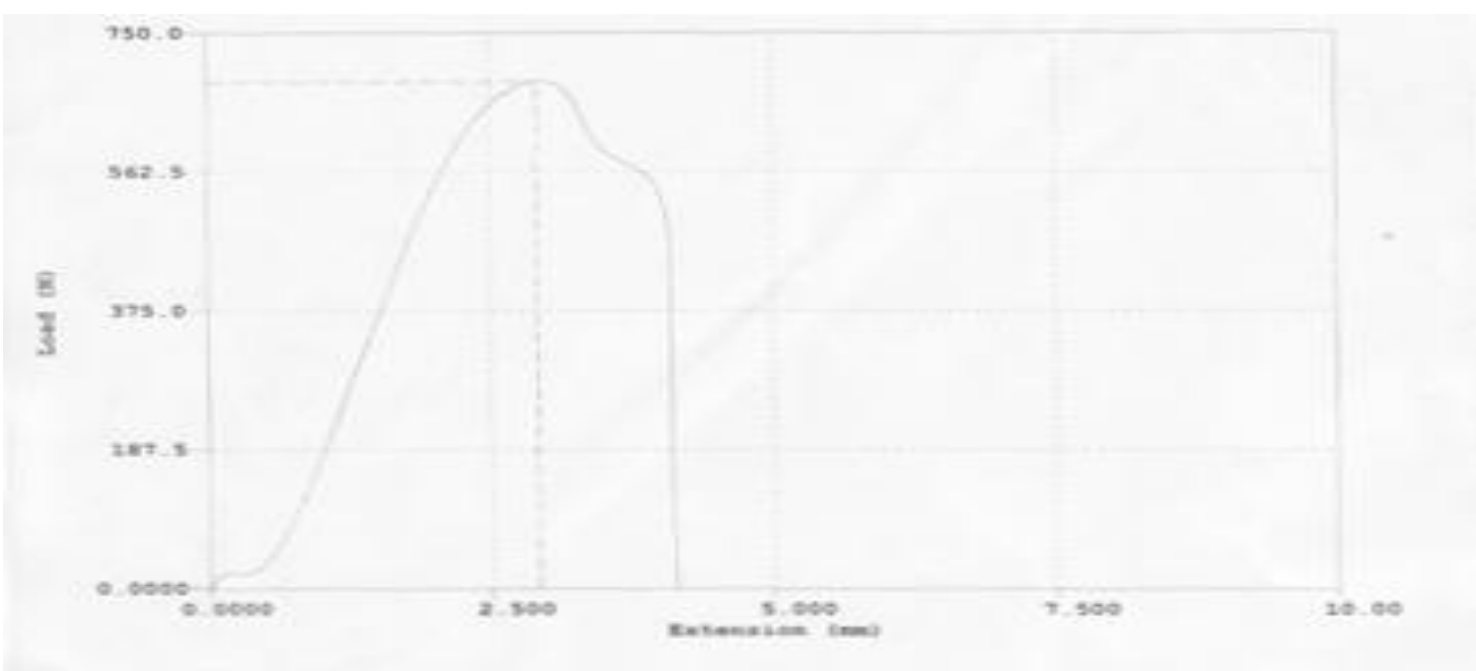

Fig. 7 The resulted load strain curve 2

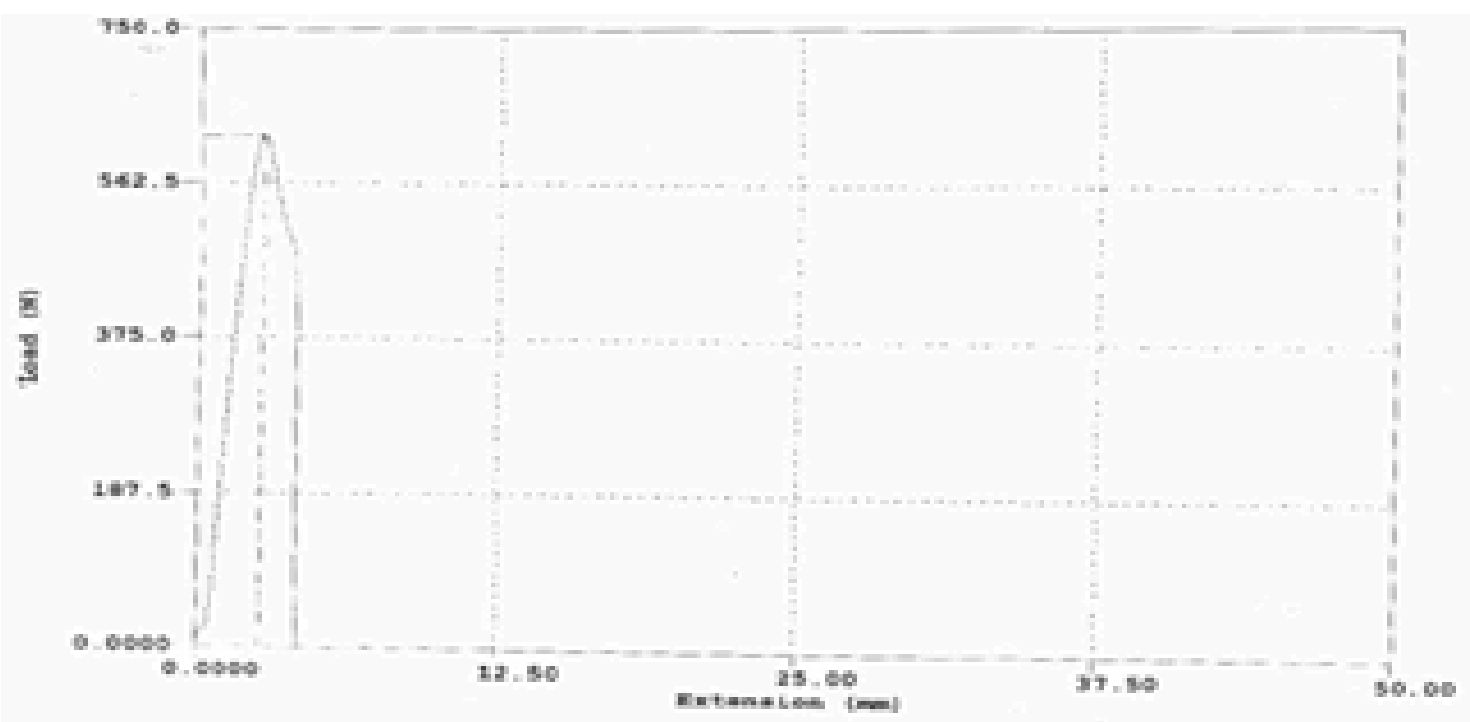

Fig. 8 The resulted load strain curve 3

From the load-displacement diagrams of the tensile tests, the ultimate tensile strength of the material (the stress to failure) was found to vary between 75 and $83 \mathrm{MPa}$.

\section{Hardness test}

Durometer Hardness (Shore Hardness-scale D) was found was found to be the suitable test for this polymeric material. The test specimens are generally $6.4 \mathrm{~mm}$ thick, in order to get the thickness, two specimens were piled together. there were cut from the base of a defected copy of the object. The test repeated five times and the results were recorded as shown in table (2)

Table 2 Hardness number values

\begin{tabular}{c|c|c|c|c|c}
\hline \hline Trial No. & 1 & 2 & 3 & 4 & 5 \\
\hline Hardness shore D & 83 & 82 & 83 & 83 & 84 \\
\hline \hline
\end{tabular}




\subsubsection{Thermal Analysis}

\section{Thermogravimetric analysis (TGA)}

This can show the thermal stability of a material, what happened to polymers when they are heated, determine weight loss of material, residuals and determine peak temperature. As shown in figure 9, the degradation occurs in the material in three steps and it remains stable till $450{ }^{\circ} \mathrm{C}$.

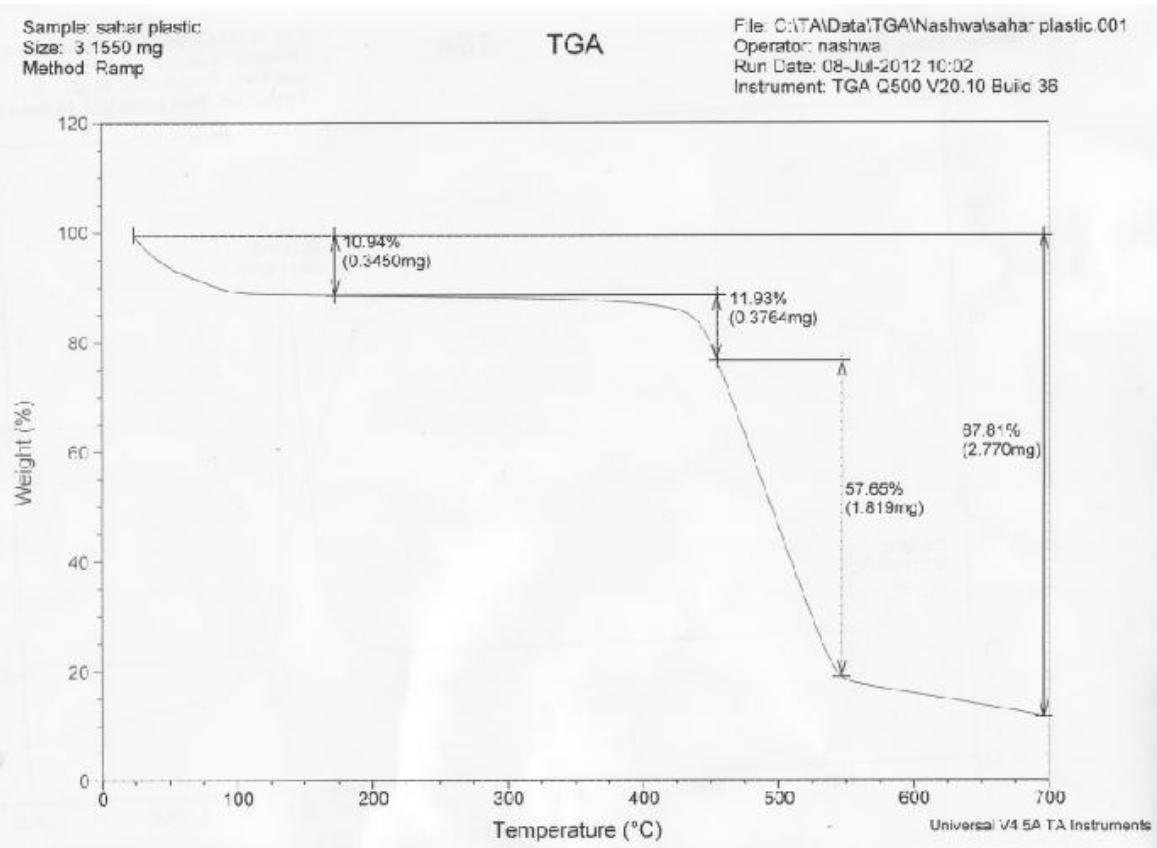

Fig. 9 TGA graph

\section{Differential scanning calorimetry (DSC)}

This test is used it to study the thermal transitions of a polymer. The thermal transitions are the changes that take place in a polymer during heating it up.

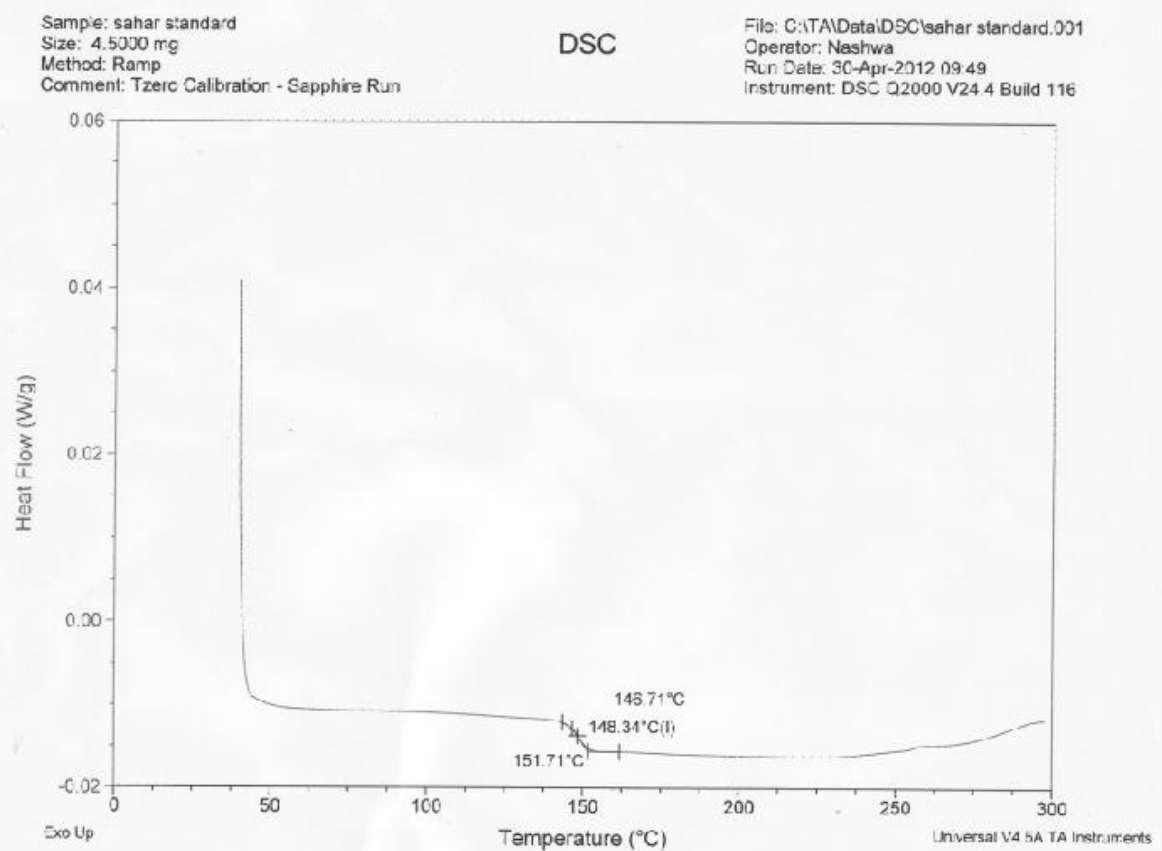

Fig. 10 DSC graph 
As shown in figure 10 the glass transition temperature $(\mathrm{Tg})$ for this material is $148.41 \mathrm{C}^{\mathrm{o}}$. And when searching on the tables of Thermal Transitions of Homopolymers it was found that polycarbonate has a $\mathrm{Tg} \approx 150 \mathrm{C}^{\circ}$. Comparing the strength, hardness and the glass transition temperature of this polymer with different polymers it was matching the polycarbonate

\subsubsection{A scanning electron microscope (SEM)}

It is used to find the composition and crystallographic information of the object material.

\section{Sample preparation}

- Sample must be of an appropriate size to fit in the specimen chamber and are generally mounted rigidly on a specimen holder called a specimen stub. So the used sample was $2 \times 4 \times 7 \mathrm{~mm}$ approximately.

- Specimens must be electrically conductive, at least at the surface, and electrically grounded to prevent the accumulation of electrostatic charge at the surface. So Nonconductive specimens tend to charge when scanned by the electron beam, this causes scanning faults and other image artifacts. Therefore specimen coated with an Aluminum coating which is electrically conducting material. Figures 11 and 12 show the resulted pictures which assured that the material of the part is polycarbonate with no additive.

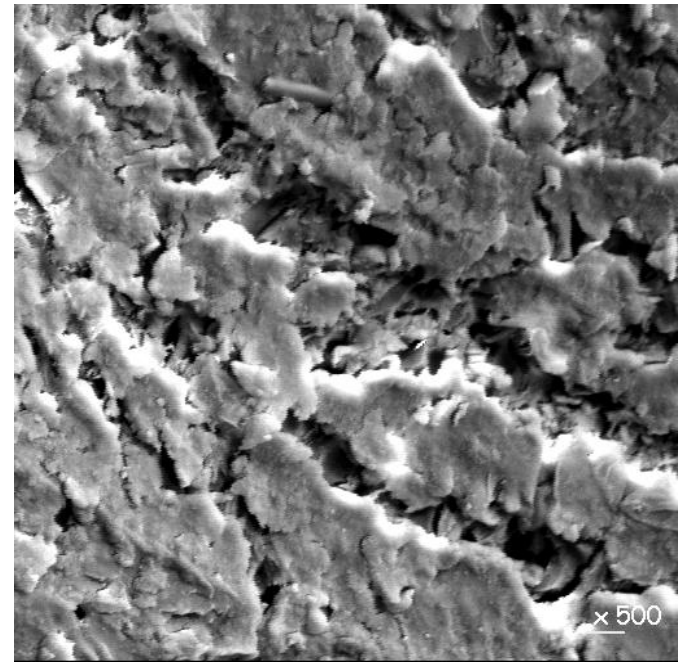

Fig. 11 SEM picture1

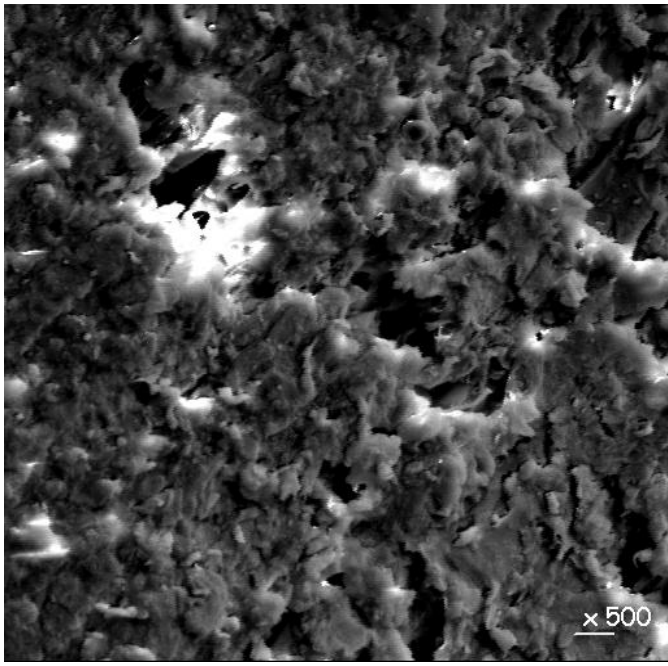

Fig. 12 SEM picture2

\subsection{Rapid Prototyping (RP) Process}

\subsubsection{Rapid proto typing procedures using SLA 5000 system}

A. Conversion of CAD model file format to STL format. Because STL (Stereolithography, the first RP technique) format has been adopted as the standard of rapid prototyping industry. This format represents a three dimensional surface as an assembly of planar triangles.

B. Slicing the STL file in this step a software program called 3D lightyear which is preprocessing program prepares the STL file to be built, this program allows the user to adjust the size, location and orientation of model. This program slices the STL model into a number of layers of $0.1 \mathrm{~mm}$ thick, as shown in figure 13. The program also generates an auxiliary structure to support the model as shown in the figure 14. 


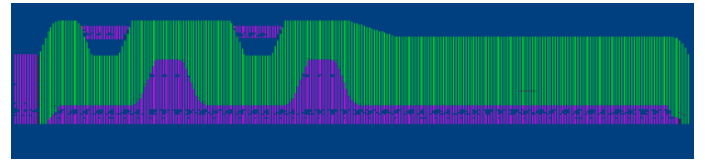

Fig. 13 Slicing the STL file

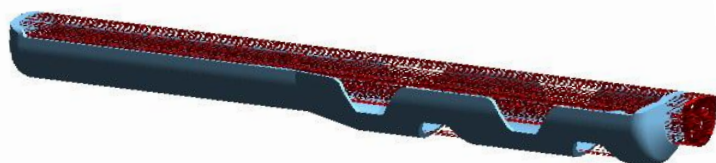

Fig. 14 Auxiliary Support

C. Layer by layer construction (construction of the part). Stereolithography is the technique used at the machine .This technique builds three-dimensional models from liquid photosensitive polymers that solidify when exposed to ultraviolet light. The model is built upon a platform situated just below the surface in a vat of liquid epoxy resin. A low-power highly focused UV laser traces out the first layer, solidifying the model's cross section while leaving excess areas liquid polymer. A sweeper re-coats the solidified layer with liquid, and the laser traces the second layer which is the top of the first and this process was repeated until the prototype part was completed.

D. Cleaning and finishing and this was the final step which is the post-processing. This involves removing the prototype from the machine and take off any supports. Figure 15 show the part from this operation.

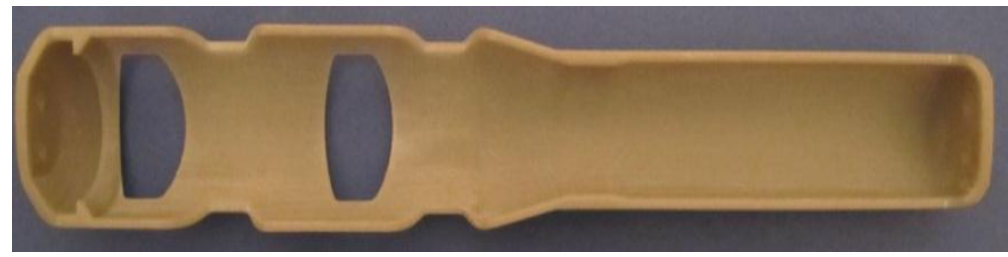

Fig. 15 The final RP part

\subsection{Comparison between the Original Part \& the RP Part}

This comparison performed using CMM by measuring the diameters of RP part at the eight sections that was measured before on the original part. The resulted diameters and its corresponding diameters are shown on Table 3.

It can be noticed that the difference between the original part measurements and the RP part is ranging between $0 \%$ to $0.7 \%$.

\section{Conclusions}

- Reverse Engineering tools were used to obtain the shape and dimensions of a mechanical part in the form of cloud of points. The need of very accurate dimensions required the use CMM measuring by probing system. This measuring means inspection of output of scanned data after converting the scanning data into a CAD file. The new technologies facilitate the reverse engineering of mechanical parts, CMM and laser scanning arms are very important tools in this aspect.

- The laser scanning alone is not capable of capturing all the details of a part especially small features and one should rely on more accurate tools like the CMM.

- NX and Geomagic software programs are very useful for reverse engineering process to obtain CAD file, Geomagic converts points cloud to polygon, NX CAD system converts surface to solid. 
- An accumulative error due to scanning, processing procedures and rapid prototyping process. But this error is very small.

- To complete the data one should determine the material and its mechanical properties to get a part with the same quality and performance of the original one.

- Rapid prototyping enables checking the results of our work before producing a real product.

Finally reverse engineering saves time, cost and risk, which has a significant effect in industrial applications.

Table 3 Comparison between the original part \& RP part

\begin{tabular}{c|c|c|c}
\hline \hline $\mathbf{Z}(\mathbf{m m})$ & $\mathbf{C M M ~ S 3}(\mathbf{m m})$ & $\mathbf{R P}(\mathbf{m m})$ & ERROR \\
\hline 44.99 & 29.125 & 29.3300 & $-0.7 \%$ \\
\hline 44.99 & 25.467 & 25.5000 & $-0.1 \%$ \\
\hline 107.9 & 29.292 & 29.3300 & $-0.1 \%$ \\
\hline 108.00 & 23.866 & 23.8000 & $0.3 \%$ \\
\hline 118.99 & 31.806 & 31.8300 & $-0.1 \%$ \\
\hline 118.99 & 27.41 & 27.5000 & $-0.3 \%$ \\
\hline 129.99 & 36.954 & 36.9000 & $0.1 \%$ \\
\hline 129.99 & 32.271 & 32.2700 & $0.0 \%$ \\
\hline 144.9 & 38.901 & 39.0000 & $-0.3 \%$ \\
\hline 145 & 34.335 & 34.3000 & $0.1 \%$ \\
\hline 187.98 & 38.109 & 38.0000 & $0.3 \%$ \\
\hline 188.00 & 34.292 & 34.3000 & $0.0 \%$ \\
\hline 202.98 & 38.492 & 38.5000 & $0.0 \%$ \\
\hline 203 & 34.202 & 34.3000 & $-0.3 \%$ \\
\hline 242.9 & 38.989 & 39.0000 & $0.0 \%$ \\
\hline 244.66 & 33.566 & 33.5805 & $0.0 \%$ \\
\hline \hline
\end{tabular}

Z: The height of the section from the base.

CMM S3: CMM measured dimensions of the best scanned copy of the part.

RP: The dimensions of the RP part.

ERROR: \% difference (in dimensions) between the best scanned original copy and the RP part.

\section{References}

[1] Manzoor Hussain M., Sambasiva Rao CH. and Prasad K. E, "Reverse engineering: point cloud generation with CMM for part modeling and error analysis", ARPN Journal of Engineering and Applied Sciences, Vol. 3, No. 4, August 2008.

[2] Ngozi Sherry Ali, “Reverse engineering of automotive parts applying laser scanning and structured light techniques ”,M Sc Thesis, The university of Tennessee Knoxville, May 2005.

[3] Vinesh Raja and Kiran J. Fernandes, “Reverse Engineering An Industrial Perspective” ,Springer, 2008.

[4] Minho Chang and Sang C. Park, "Reverse engineering of a symmetric object" ScienceDirect, Computer \& Industrial Engineering, 2008.

[5] D. K. Pal, Dr. B. Ravi 1, L. S. Bhargava, "Computer-Aided Reverse Engineering for Rapid Replacement Parts: A Case Study”, Defence Science Journal, DESSIDOC, DRDO, New Delhi, 2005. 
[6] http://en.wikipedia.org/wiki/Reverse_engineering, “Reverse engineering”,February.,2013.

[7] Ingle, Kathryn A., "Reverse Engineering”, McGraw-Hill, Inc., 1994, pages1-35.

[8] Gudimetal P. and Gopinath C.V., "Finite Element Analysis of Reverse Engineered Internal Combustion Engine Piston”, AIJSTPME, Vol.2, No.4, 2009, pages 85-92.

[9] ManzoorHussain M., SambasivaRao CH. and Prasad K. E, “Reverse engineering: point cloud generation with CMM for part modeling and error analysis, ”ARPN Journal of Engineering and Applied Sciences, Vol. 3, No. 4, August 2008.

[10] Y.H. Chen and C.T. Ng, "Integrated Reverse Engineering and Rapid Prototyping", Computers ind, Engng, Vol. 33, Nos. 3-4, 1997, pages 481-484.

[11] Basilio Ramos Barbero, Elena Santos Ureta, "Comparative study of different digitization techniques and their accuracy", Science Direct Computer-Aided Design, Vol.43, 2011, Pages 188-206. 